\title{
Advanced Mechatronics Monitoring and Control of Spatially Distributed Systems
}


This page intentionally left blank 


\section{Advanced Mechatronics Monitoring and Control of Spatially Distributed Systems}

\section{Dan Necsulescu}

University of Ottawa, Canada 
Published by

World Scientific Publishing Co. Pte. Ltd.

5 Toh Tuck Link, Singapore 596224

USA office: 27 Warren Street, Suite 401-402, Hackensack, NJ 07601

UK office: 57 Shelton Street, Covent Garden, London WC2H 9HE

\title{
British Library Cataloguing-in-Publication Data
}

A catalogue record for this book is available from the British Library.

\begin{abstract}
ADVANCED MECHATRONICS
Monitoring and Control of Spatially Distributed Systems

Copyright $(\subseteq) 2009$ by World Scientific Publishing Co. Pte. Ltd.

All rights reserved. This book, or parts thereof, may not be reproduced in any form or by any means, electronic or mechanical, including photocopying, recording or any information storage and retrieval system now known or to be invented, without written permission from the Publisher.
\end{abstract}

For photocopying of material in this volume, please pay a copying fee through the Copyright Clearance Center, Inc., 222 Rosewood Drive, Danvers, MA 01923, USA. In this case permission to photocopy is not required from the publisher.

ISBN-13 978-981-277-181-0

ISBN-10 981-277-181-6

Printed in Singapore. 
To Cristina, Philip and Roxana 
This page intentionally left blank 


\section{Preface}

Mechatronics is an engineering field that refers to mixed systems tight integration. Currently, this integration can be viewed as based on digital computer monitoring and control, but it cannot be denied that integration can be based on any other signal processing system and any form of raw power that can be modulated and transferred to the mixed system in accordance to the output of this digital signal processor.

Distributed parameters systems, in the form of solids, liquids, gases, are seen as fields in which the dynamics can be represented by traveling waves. These fields can be mechanical vibration fields of substance, acoustic, electromagnetic etc. The assumption of continuity is often limited, when moving down from macroscopic level, by the molecular, atomic discontinuous structure, which can be represented in duality with the fields at that level. Moving up from immediate macroscopic level towards infinite celestial level, again the continuum of the quasi-vacuum space is filled with solid planets etc. Consequently, while at terrestrial macroscopic level, continuous fields can be assumed of infinite dimensions, there are perceived limits as we move up and down from this level.

Distributed Parameters Systems are modeled mathematically by partial differential equations and/or multiple integrals that can be recast also in a system of partial differential equations. The solutions of these partial differential equations show that the dynamics of distributed parameters systems can be simulated as composed of infinite dimensional combinations of harmonic components (something that might remind of Pythagoras' view of the planets motions) where higher frequency components might become less and less significant unless excited and brought to resonance. 
Mechatronics refers to monitoring, control and integration not only of lumped parameters systems, but also of distributed parameters systems. In fact, the latter representation of the world under engineering focus is more realistically modeled by distributed parameters systems; handling such models is, however, much more difficult that the lumped parameters systems. Monitoring and control of distributed parameters systems is limited by ill-posed problems, the inverse problems of estimating system states and parameters from sensors signals and controlling and infinite dimensional system with modulated power output from actuators. Sensors and actuators are available in most cases as point devices and, even if they are distributed, they cannot be found in the infinite dimensional form. Sensors and actuators are bandwidth limited and cannot access higher frequency components of distributed parameters systems dynamics. As a result, only lower frequency dynamics can be controlled and maybe somewhat higher but still low frequency components can be monitored; higher frequency dynamics remains uncontrolled and unobserved. Pascal made a valid comment with regard to human condition in an infinite world: “...qu'est que l'homme dans la nature? Un néant à l'égard de l'infini, un tout à l'égard du néant, un milieu entre rien et tout." (B. Pascal, Pensées, no. 72). Using science and engineering, we reach easily documented limits in monitoring and controlling such systems and only religion, art and philosophy can offer further views outside these limits. Indeed, direct view, i.e. intuitive access to that level requires to become detached from contact and affection from the immediate and finite environment and to bring ourselves to the vision of infinite spaces.

I acknowledge the results documented in the book of joint published research with my colleagues professors Dr. R. Baican F. BakhtiariNejad, J. Sasiadek and W. Weiss and with my former graduate students: R. F. De Abreu, G. M. Ceru, G. Ganapathy, Kuoc-Vai Iong, Y. Jiang and W. Zhang. 


\section{Contents}

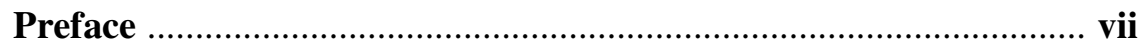

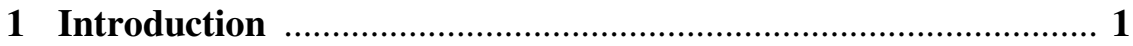

1.1 Advanced Mechatronics Systems. Monitoring and Control of Distributed Parameters Systems ......................................... 1

1.2 Signals versus Power Transmission. Lumped Parameters Modeling of Mechatronic Systems …….................................. 3

1.2.1 Effort flow variables and two port models ..................... 6

1.2.2 Newton-Euler and Kirchhoff equations for a mixed electro-mechanical system ........................................ 10

1.2.3 Lagrange equations for a mixed electro-mechanical system ............................................................... 14

1.3 Local Sensing and Actuation in Spatially Continuous Systems 30

1.3.1 Lumped parameters models with under-actuation and under-sensing

1.3.2 Distributed parameters models with under-actuation

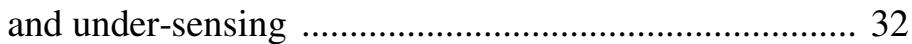

1.4 Centralized versus Local Control ............................................... 32

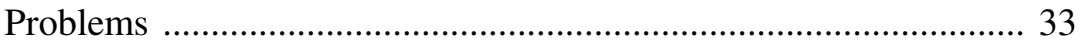

\section{Examples of Direct and Inverse Problems for Mixed} Systems

2.1 Modular Modeling and Control Issues for Mixed Systems 35 2.1.1 Effort-flow modeling of mechatronic systems 35 
2.2 Modeling and Simulation of Distributed Parameters

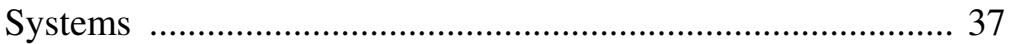

2.2.1 Examples of distributed parameters systems ................ 37

2.2.1.1 Examples of models of vibrating flexible structures ......................................................... 37

2.2.1.2 Acoustic fields .............................................. 40

2.2.1.3 Heat transfer ................................................ 41

2.2.1.4 Fluid flow ....................................................... 41

2.2.1.5 Electric and magnetic fields ............................. 42

2.2.2 Direct and inverse problems. Well posed and ill posed problems ........................................................ 43

2.2.3 Classification of partial differential equations and methods of solving

2.3 Overview of Open Loop and Closed Loop Control of

Distributed Parameters Systems 46

2.3.1 Direct and inverse problems ....................................... 46

2.3.2 Inverse heat conduction problem ................................. 48

2.3.3 Open loop control of distributed parameters systems ............................................................... 51

2.3.4 Closed loop control of distributed parameters

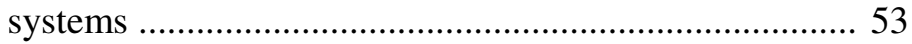

2.4 Under-Actuated and Under-Sensed Mixed Systems ............... 54 2.4.1 General problem of multi DOF linear mechanical systems. Lumped parameters model ........... 54

2.4.2 Two DOF mechanical system case ........................... 55

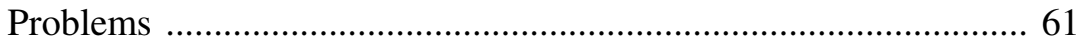

\section{Overview of Integral Equations and Discrete Inverse}

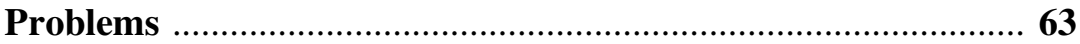

3.1 Integral Equations and Continuous Inverse Problems ............ 63

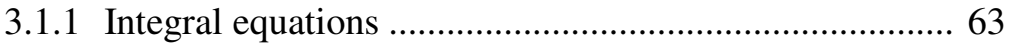

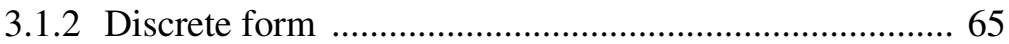

3.1.3 Other examples of discrete inverse problems ............... 67

3.2 Discrete Problems for LTI Systems …….............................. 70

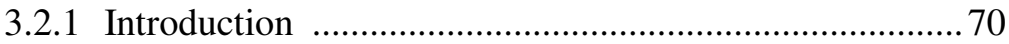


3.2.2 Lumped parameters systems ....................................... 71

3.2.2.1 State space representation ............................. 71

3.2.2.2 Complex functions representation .................. 72

3.2.2.3 Convolution integral representation ................ 73

3.2.2.4 Matrix form representation ............................ 80

3.3 Discrete Inverse Problems Solved by Matrix Inversion ........... 91

3.3.1 Types of methods for solving inverse problems ........... 91

3.3.2 Inverse and pseudo-inverse. MATLAB solutions ......... 94

3.3.3 Over-determined and under-determined problems ...... 104

3.3.4 SVD method .......................................................... 113

3.3.5 Damped LS solution .............................................. 119

3.3.6 Regularization method. Regularized LSS .................. 119

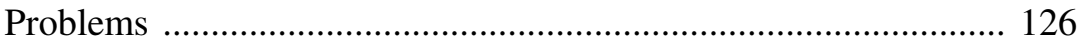

4 Inverse Problems in Dynamic Calibration of Sensors ............. 129

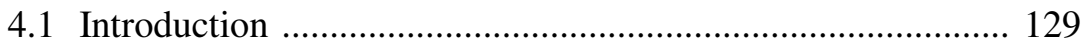

4.2 First Order Instruments .................................................... 130

4.2.1 Time and frequency response of forward

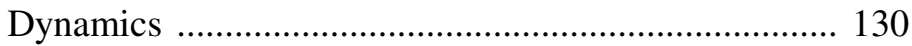

4.2.2 Bandwidth of first order instruments ....................... 132

4.2.3 Static calibration of the sensor ................................. 132

4.2.4 Sinusoidal response of the sensor -

MATLAB simulations ............................................. 134

4.2.5 Analytical solutions for harmonic response of first order instruments .............................................. 137

4.3 Second Order Instruments ................................................. 140

4.3.1 Static calibration ......................................................... 140

4.3.2 Harmonic response of the second order sensor with $\zeta=0.6$. MATLAB simulations .......................... 148

4.3.3 Analytical solutions for harmonic response of a second order instrument ........................................... 151

4.4 Calibration for Computer-Based Instrumentation ................. 156

4.4.1 Calibration for computer based first order instruments

4.4.2 Phase lead compensation 
4.4.3 Full and reduced order dynamic compensators ........... 166

4.4.3.1 First order instrument .................................. 169

4.4.3.2 Second order instrument .............................. 170

4.5 Dynamic Calibration in Case of Noisy Measurements .......... 173

4.6 State Estimation for Indirect Sensing ................................. 179

4.6.1 Derivation of the estimator for indirect states estimation using matrix inversion approach ............... 179

4.6.2 Luenberger observers and Kalman filters ................... 183

4.6.3 Indirect estimation of states and inputs for

LTI ODE systems using matrix inversion .................. 185

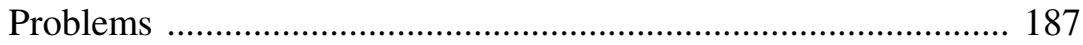

5 Active Vibration Control in Flexible Structures .................... 189

5.1 Active Vibration Suppression for Lumped Parameters

Mechanical Systems Using Force and Position Control ........ 189

5.1.1 Direct problem ......................................................... 189

5.1.2 Force control for SISO mechanical system ................ 192

5.1.3 Position feedback control approach ............................ 194

5.2 Direct Problem and Under-Actuated Control of a

Non-Minimum Phase Flexible Shaft ................................... 197

5.3 Control of Vibrations in Beams .......................................... 202

5.3.1 Perturbation cancellation control in MIMO

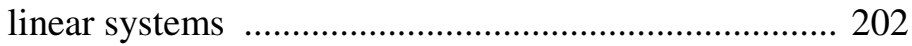

5.3.2 Direct problem in beam vibration modeling ............... 206

5.3.3 Feedback control of transversal vibrations in beams ... 210

5.3.4 Feedback modal control ........................................ 216

5.3.5 Modal control in beam vibration ................................ 224

5.4 Direct Problem in Free Vibrations in Membranes ................ 225

5.4.1 Membrane vibration solution plotting ....................... 227

5.4.2 Simulation of membrane using FEMLAB ................. 230

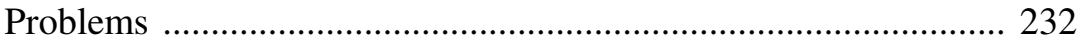

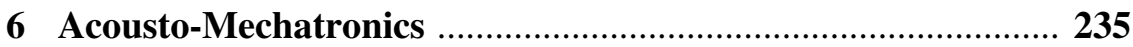

6.1 Acousto-Mechatronic Systems ........................................ 235 
6.1.1 Recording studio ....................................................... 235

6.1.2 Active sound control in halls .................................. 236

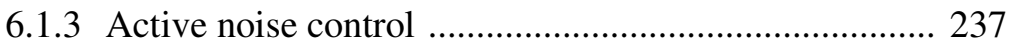

6.2 Distributed Parameters Models of Sound Transmission ........ 238

6.2.1 Wave equation for planer sound wave 1D propagation in a free sound field ................................................ 238

6.2.2 Wave equation for planar sound wave 3D propagation a free sound field

6.2.3 Sound wave propagation in an enclosed sound field

6.3 Calculation of Eigenvalues and Eigenvectors for a Rectangular Cavity ........................................................ 246

6.4 Experimental and Simulation Study of Room Acoustics ....... 254

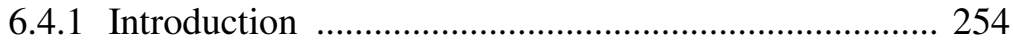

6.4.2 Proposed approach ................................................. 255

6.4.3 Simulation model ................................................ 256

6.4.4 Simulation results based on ray propagation approach .......................................................... 258

6.4.5 Experimental results ................................................ 260

6.5 Discrete Inverse Problems based on Direct and Reflected

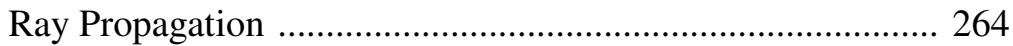

6.5.1 Parameters estimation using direct ray propagation .... 264

6.5.2 Other inverse problems using ray propagation ............ 271

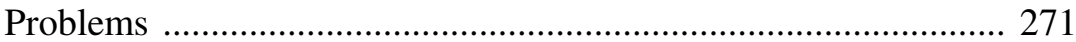

7 Themo-Mechatronics ……………………………………............. 273

7.1 Direct Problem: Heat Flow Modeling and Simulation ........... 273 7.1.1 Direct problem solving for 2-Dimentional (2D) heat conduction from a distributed heat source ................... 273

7.1.2 Direct problem simulation of $2 \mathrm{D}$ heat flow for a continuous point-heat source input using MAPLE $^{\mathrm{TM}}$

7.1.3 Simulation of $2 \mathrm{D}$ heat flow for a short temperature pulse input using FEMLAB ${ }^{\mathrm{TM}}$ 278

7.1.4 Direct problem formulation for 3-D heat flow 282 
7.2 Inverse Problem Solution for Remote Temperature

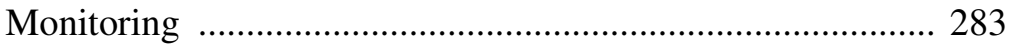

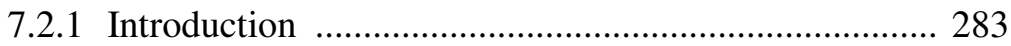

7.2.2 Inverse problem for heat flux input remote estimation from temperature measurements ............... 284

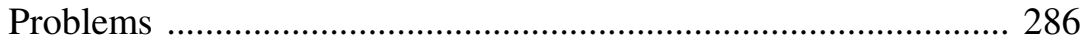

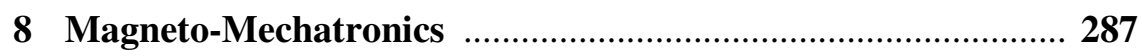

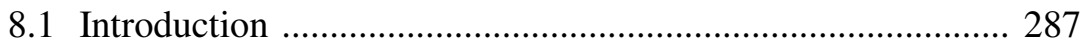

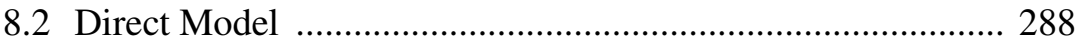

8.3 Simulation Results for Linear Control ................................. 290

8.4 State-Input Linearization of a Magnetic

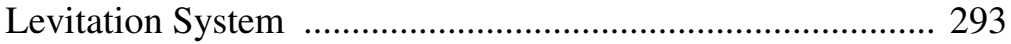

8.4.1 Feedback linearization ............................................. 293

8.4.2 State-Input linearization and linear

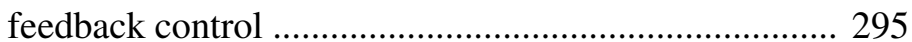

8.5 Nonlinear Controller of a Magnetic Suspension System ....... 297

Problems ................................................................................ 300

9 Inverse Problems Issues for Non-Minimum Phase Systems .... 301

9.1 Direct and Inverse Problems for Non-Minimum Phase Nonlinear Systems .......................................................... 301

9.1.1 Introduction ............................................................ 301

9.1.2 Direct problem for non-minimum phase systems ........ 301

9.1.3 Neural network approach to inverse dynamics ........... 303

9.2 Feedback Linearization of a Non-Minimum Phase UAV ........303

9.3 Mathematical Model for UAV Direct Problem ...................... 305

9.4 Simulation Results for the Neural Controller and Output Redefinition ........................................................................... 312

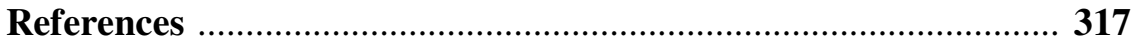

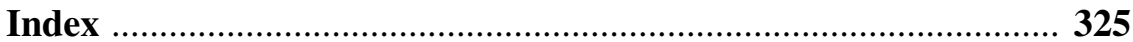

